Revue internationale P.M.E.

Économie et gestion de la petite et moyenne entreprise

\title{
Internet, facteur de gains de productivité et de diversification dans les PME : caractérisation des contextes d'usage
}

\section{Serge Amabile et Martine Gadille}

Volume 15, numéro 3-4, 2002

URI : https://id.erudit.org/iderudit/1008815ar

DOI : https://doi.org/10.7202/1008815ar

Aller au sommaire du numéro

Éditeur(s)

Presses de l’Université du Québec

ISSN

0776-5436 (imprimé)

1918-9699 (numérique)

Découvrir la revue

Citer cet article

Amabile, S. \& Gadille, M. (2002). Internet, facteur de gains de productivité et de diversification dans les PME : caractérisation des contextes d'usage. Revue internationale P.M.E., 15(3-4), 115-138. https://doi.org/10.7202/1008815ar

\section{Résumé de l'article}

Notre recherche s'intéresse aux caractéristiques de PME ayant simultanément su atteindre des gains de productivité et mettre en œuvre des processus de diversification grâce à leurs usages d'Internet. Dans un environnement économique en évolution permanente, marqué par l'innovation et la concurrence, cette double capacité apparaît comme un élément important de la viabilité et de la pérennité des petites et moyennes structures pour lesquelles la diversification externe, pratiquée par les grandes organisations, correspond à une stratégie trop coûteuse et donc, dans la pratique, inaccessible. La capacité d'obtention de combinaisons de ces deux avantages concurrentiels grâce aux usages d'Internet et de ses technologies connexes est expliquée par une analyse statistique détaillée des usages de la technologie reconsidérés dans leurs contextes organisationnels et industriels. 


\title{
Internet, facteur de gains de productivité et de diversification dans les PME : caractérisation des contextes d'usage
}

\author{
Serge AMABILE \\ Université d'Aix-Marseille III
}

Martine GADILLE1

Centre national de la recherche scientifique

\section{MOTS CLÉS}

\section{PME - Internet - Avantage concurrentiel - Productivité Diversification - Contexte d'usage - Espace industriel}

1. L'ordre de signature des auteurs respecte simplement l'ordre alphabétique.

\section{LES AUTEURS}

SERGE AMABILE est membre du conseil pédagogique du DEA en «Ingénierie des organisations socioéconomiques » du GREQAM (UMR CNRS 6569), coordinateur des enseignements en gestion de l'IUP Miage d'Aix-en-Provence. Ses recherches portent principalement sur le management des technologies de l'information dans les organisations et sur l'ingénierie des systèmes d'information de veille stratégique ; sur ces thèmes, il est auteur de plusieurs publications dans différentes revues (Systèmes d'information et management, Revue française de gestion, Sciences de la société). Adresse: GREQAM (UMR CNRS) - Faculté d'économie appliquée - Université d'Aix-Marseille III, 15, 19, allée C.-Forbin, 13627 Aix-en-Provence Cedex. Courriel : serge.amabile@univ.u-3mrs.fr

MARTINE GADILLE mène des recherches sur les mécanismes de diffusion d'Internet dans les PME selon une approche basée sur l'appropriation et le développement par l'usage, participant à des processus d'innovation dans des espaces industriels spécifiques. Les principales revues qui ont accueilli ses publications sont les suivantes: Economie appliquée, Réseaux, RFG (en cours de publication), Systèmes d'information et management, Travail et emploi, Formation emploi. Adresse: Institut de droit et d'économie de la firme (IDEFI) - LATAPSES (UMR CNRS-Université de Nice), 1, rue A.-Einstein, Sophia Antipolis, 06560 Valbonne. Courriel : gadille@univ-aix.fr 


\title{
RÉSUMÉ
}

Notre recherche s'intéresse aux caractéristiques de PME ayant simultanément su atteindre des gains de productivité et mettre en œuvre des processus de diversification grâce à leurs usages d'Internet. Dans un environnement économique en évolution permanente, marqué par l'innovation et la concurrence, cette double capacité apparaît comme un élément important de la viabilité et de la pérennité des petites et moyennes structures pour lesquelles la diversification externe, pratiquée par les grandes organisations, correspond à une stratégie trop coûteuse et donc, dans la pratique, inaccessible. La capacité d'obtention de combinaisons de ces deux avantages concurrentiels grâce aux usages d'Internet et de ses technologies connexes est expliquée par une analyse statistique détaillée des usages de la technologie reconsidérés dans leurs contextes organisationnels et industriels.

\begin{abstract}
The results presented in this paper deal with the characteristics of SME's capable to benefit, on the bases of their Internet uses, from productivity gains and diversification practices. That double capacity appears to be in a flexible and highly competitive economy as an important mean of viability for SME's compared to big firms which are able to survive by external diversification. The capacity to obtain simultaneously that two sources of competitive advantage is explained by a precise statistical analysis of the technology uses that are reconsidered in their organizational and industrial contexts.
\end{abstract}

\section{RESUMEN}

Nuestro informe se interesa en las características de las pequeñas y medias empresas que han alcanzado ganancias de productividad y que han al mismo tiempo puesto en marcha los procesos de diversificación gracias a sus usos del red. En un medioambiente económico en permanente evolución, marcado por la innovación y la competencia, ese doble capacidad aparece como un elemento importante de la viabilidad y de la perennidad de las pequeñas y medias estructuras. Por estas últimas empresas, la diversificación externa practicáda por las grandes firmas más corresponde a una estrategia demasiado costosa que se vuelve, por entonces en la práctica, inaccesible para ellas. La capacidad de obtención de la combinación de esos dos ventajas de competencia, gracias al red y a sus tecnologías conexas, está explicada por un análisis estadístico detallado de los usos de la tecnología pero repuestos en sus contextos de organización e industriales.

\section{ZUSAMMENFASSUNG}

Unsere Untersuchung interessiert sich für KMU, die gleichzeitig Produktivitätsgewinne realisiert und Diversifikationsprozesse auf die Beine gestellt haben dank der Nutzung des Internets. In einem ökonomischen Umfeld mit einer ständigen Entwicklung, gekennzeichnet durch Innovation und Konkurrenz, erscheint diese zweifache Fähigkeit als ein wichtiges Element für die Lebensfähigkeit und den Fortbestand der kleinen und mittleren Strukturen. Normalerweise ist die externe Diversifikation, wie sie angewendet wird durch grosse Organisationen, eine zu teure 
und unerreichbare Strategie für KMU. Durch die Nutzung des Internets und seinen verwandten Technologien können zwei Konkurrenzvorteile kombiniert werden. Dies wird in der Folge erklärt durch eine detaillierte statistische Analyse der Technologienutzung in ihrem organisatorischen und industriellen Kontext.

\section{Introduction}

La diffusion massive d'Internet et de l'usage du Web conduit à reconsidérer les effets des technologies de l'information et de la communication (TIC) sur les conditions ou les contextes d'obtention d'avantages concurrentiels ou, plus simplement, sur la viabilité de l'entreprise et notamment de la PME. En effet, Internet et le Web peuvent représenter pour les PME un palliatif de surfaces financières mobilisables (pour la mise en œuvre de processus de prospection de nouveaux marchés, de nouveaux fournisseurs, partenaires, etc.) a priori plus « modeste » que dans les grandes organisations. De fait, avec l'apparition massive de cette technologie, la productivité et les coûts ne semblent plus être les principaux motifs ni les seuls critères de production d'avantages concurrentiels à l'aune desquels les retours sur l'investissement dans l'usage de TIC peuvent être mesurés.

Ainsi, depuis cinq ans, nous avons tiré de nos recherches deux enseignements principaux : d'une part, l'utilisation d'Internet favorise, sous certaines conditions, la réalisation de gains de productivité, la réduction des coûts, le raccourcissement des délais de production et de transaction ou, encore, l'amélioration de la qualité ; d'autre part, elle peut favoriser l'apparition de processus de diversification de produits ou services pour les PME «ordinaires» (Gadille et Amabile, 2002). Reposant sur une capacité de compréhension, d'adaptation, voire de création de nouveaux marchés, ces processus de diversification permettent à l'entreprise de se différencier de la concurrence sur un secteur en particulier. Sans pour autant exiger des investissements importants dans des progiciels de gestion de l'information, les TIC fondées sur les protocoles de l'Internet peuvent, en effet, être mobilisées comme instruments de veille économique, technologique et législative, mais aussi comme des outils de gestion commerciale et de suivi de la clientèle. Par exemple, l'usage de l'Internet et, plus particulièrement, du Web permet d'accéder à des informations auxquelles l'entreprise pouvait difficilement parvenir auparavant ou, plus simplement, développe l'accessibilité de multiples documents tels que les textes législatifs, les normes, les règlements, etc. Ainsi, l'utilisation d'un site, une activité de veille économique ou l'observation des offres des fournisseurs sur le Web peuvent être les premiers supports de la diversification de la PME. Or, pour une petite structure, cette diversification est plus viable lorsqu'elle reste associée à des gains de productivité. De fait, il semble intéressant d'appréhender les caractéristiques

Revue internationale P.M.E., vol. 15, $\mathrm{n}^{\text {os }} 3-4,2002$ 
des PME, capables simultanément, à partir de leurs usages d'Internet, de se diversifier vers de nouveaux produits ou services et d'obtenir des gains de productivité grâce à cette technologie (Amabile et Gadille, 2003).

Ces deux sources d'avantages concurrentiels peuvent résulter de stratégies génériques (Porter, 1985). Elles s'inscrivent non seulement dans des aspects d'organisation et de pratiques professionnelles héritées du passé (March, 1991), mais aussi dans l'espace industriel de cette entreprise, c'est-à-dire dans les interdépendances et les interactions que l'entreprise entretient avec ses fournisseurs, partenaires, concurrents et avec d'autres types d'acteurs (privés ou publics) de son environnement. Ce sont donc ces trois dimensions qui ont été investies dans notre dispositif d'enquête afin d'appréhender la capacité de valorisation de la technologie selon les deux critères mobilisés dans cette introduction, à savoir les gains de productivité et les processus de diversification.

Une première section offre d'abord une présentation rapide de la théorie relative à la problématique des usages, puis, du questionnement mis en œuvre dans l'enquête statistique dont on exploite les résultats. Les sections suivantes présentent les caractéristiques d'une population de PME qui innove et réalise des gains de productivité à partir de ses usages d'Internet en les comparant avec les PME d'une deuxième population qui n'obtient aucun de ces deux impacts. Sont ainsi analysées les différences en matière d'équipements en TIC et en réseaux à valeur ajoutée, les divergences en matière de capacités professionnelles et organisationnelles et, finalement, les différences de modalités d'insertion dans l'espace industriel.

\section{Une approche fondée sur les usages construits dans des espaces organisationnels et industriels}

En général, les approches standard de l'innovation technologique (Nelson et Winter, 1982) en économie de l'innovation se préoccupent plus de l'étude des processus conduisant à produire un nouveau bien ou service que de l'étude du contexte d'appropriation de cette innovation au sein des entreprises concernées. Néanmoins, certains analystes du changement technologique ont étudié la continuité du processus d'innovation à l'intérieur des organisations. Ainsi, Rosenberg (1982) met en évidence l'idée d'apprentissage par l'usage. Les observations et les interprétations de cet auteur convergent vers la théorie du changement dans les organisations et le concept d'apprentissage organisationnel (Levitt et March, 1988). Elles se rapprochent également de représentations plus « sociétales » mettant en évidence le lien entre, d'une part, la configuration des usages de la technologie et les capacités professionnelles et organisationnelles de l'entreprise et, d'autre part, la configuration de l'espace industriel dans lequel celle-ci est insérée (Eyraud, d'Iribarne et Maurice, 1988). De tels travaux permettent de fonder la notion d'appropriation de

Revue internationale P.M.E., vol. 15, ns 3-4, 2002 
la technologie posée en contrepoint de l'approche diffusionniste (Rogers, 1983; Gadille et d'Iribarne, 2000b). Ils offrent des outils conceptuels pour expliciter les contextes d'usages favorables ou non à la production d'avantages concurrentiels.

\subsection{Modes d'usages de la technologie et sources d'avantages concurrentiels}

Les sources d'avantages concurrentiels s'appuient sur des systèmes techniques d'information et de communication qui constituent aujourd'hui un élément essentiel de la compétitivité. Ces systèmes sont utilisés selon des modalités d'usages qui relèvent, dans une certaine mesure, de stratégies mais aussi de routines des responsables de l'entreprise et des utilisateurs. Ces usages peuvent mettre en relation des partenaires à l'intérieur ou à l'extérieur de l'entité organisationnelle considérée, selon des applications et des domaines d'activité divers (ce que nous préciserons par la suite). Les modalités d'utilisation du système d'information et de communication sont dépendantes des capacités organisationnelles et collectives d'appropriation des technologies (Gadille et d'Iribarne, 2000a) qui sont elles-mêmes transformées dans le temps par les interactions entre différentes stratégies d'usage et de développement d'applications, dont une part relève de résultats non intentionnels.

\subsection{L'espace professionnel, une notion pour appréhender les conditions d'un apprentissage satisfaisant}

Les possibilités de développement et d'usages des TIC liées à Internet sont peut-être moins liées à leurs coûts d'achat et de développement qu'au manque d'apprentissage ou, simplement, de connaissance des usages possibles de ces technologies. Les capacités d'apprentissage sont définies par des combinaisons entre des processus d'exploration et d'exploitation de routines (March, 1991). S'agissant des usages d'Internet, ces processus entretiennent une relation d'interdépendance dans le temps: la recherche de nouvelles règles d'usage se fait sur les bases d'anciennes et se traduit dans le temps par de nouvelles routines. Ces interactions se concrétisent par l'évolution des compétences et des connaissances de divers acteurs supportant de nouvelles possibilités d'usages ou de nouvelles possibilités technologiques (Gadille et d'Iribarne, 2000c). Cette évolution peut, elle-même, influencer le processus de prise de décision stratégique à partir des compétences, des connaissances et, plus largement, des « cadres interprétatifs » du management (Amabile et Gadille, 2003).

En ce sens, l'apprentissage par l'usage permet de faire évoluer les cadres cognitifs de la prise de décision ainsi que les compétences mises en œuvre. Ainsi, les choix stratégiques restent médiatisés et dépendant des «capacités professionnelles » que l'entreprise a pu construire à partir de sa trajectoire passée. La notion de capacités professionnelles permet d'appréhender les notions de «capacités

Revue internationale P.M.E., vol. 15, nºs 3-4, 2002 
organisationnelles » ou « capacités dynamiques » telles qu'elles sont conceptualisées par des auteurs évolutionnistes (Chandler, 1992; Nelson, 1991; Teece et Pisano, 1998). Les avantages concurrentiels que peut obtenir une entreprise au moyen de nouvelles technologies ${ }^{2}$ sont donc liés, pour partie, au phénomène d'apprentissage par l'usage de ces technologies (Rosenberg, 1982) sur la base de ses capacités professionnelles et organisationnelles.

\subsection{La trajectoire technologique comme produit de l'insertion dans un espace industriel}

Les auteurs évolutionnistes partent du postulat de la nature cumulative et dynamique du changement technique (Nelson et Winter, 1982). Les ensembles de routines résultent, de façon originale, de l'expérience acquise par l'entreprise sur son marché, ce qui explique que certaines entreprises survivent plus facilement compte tenu de leur trajectoire initiale. Nelson et Winter ont ainsi fait émerger la notion de « trajectoires technologiques » pour signifier que les cheminements du développement technique dans une organisation sont cumulatifs, auto-créateurs et s'autonomisent de toute référence répétée à l'environnement économique externe à l'entreprise. Selon cette même perspective, les processus et les produits d'une entreprise sont difficiles à imiter en raison des connaissances technologiques et des compétences de la firme. C'est le caractère tacite d'une partie de la connaissance technologique qui permet de soutenir l'hypothèse d'un progrès technique local et cumulatif, d'où la place accordée au concept d'apprentissage dans le processus d'évolution exprimé par la notion de trajectoire technologique. Néanmoins, l'aspect local et cumulatif de la technologie et des compétences reste lié aux caractéristiques des relations que l'entreprise entretient dans son «espace industriel» (Gadille et d'Iribarne, 2000 ) comme, par exemple, son insertion dans divers réseaux d'entreprises. C'est dans cet espace industriel que se nouent des types de relations entre différentes activités marchandes ou non marchandes, de nature industrielle et concurrentielle, technologique ou scientifique, financière ou administrative. Ces relations peuvent être le support d'interactions contribuant au développement de la capacité d'adaptation de l'entreprise ou, en d'autres termes, à des processus d'apprentissage organisationnel (Levitt et March, 1988).

La mobilisation d'Internet et des technologies qui lui sont connexes afin de médiatiser les relations de l'organisation avec différents types de «partenaires externes » peut alors être à la fois perçue comme un révélateur des stratégies d'usages et des possibilités de capter des externalités de réseaux agissant sur les capacités professionnelles et organisationnelles.

2. Précisons qu'à travers l'expression «nouvelles technologies » Rosenberg ne désignait pas uniquement les TIC même si celles-ci sont intégrées dans ses développements. 


\subsection{Méthode}

Afin de mieux connaître les caractéristiques des entreprises capables, sur la base de leurs routines, de leur stratégie et de leurs usages d'Internet, de réaliser des gains de productivité et, simultanément, de mettre en œuvre un processus de diversification, des questions directes ont été posées aux responsables pour connaître leur opinion sur:

- l'effet d'Internet sur la productivité;

- l'effet d'Internet sur la diversification vers de nouveaux produits ou services.

À partir des réponses, deux grandes catégories de PME ont été établies afin de procéder à une première analyse comparative :

- la première population regroupe les 183 entreprises dont les responsables ont constaté un double effet positif de l'usage d'Internet à la fois sur les gains de productivité et la diversification; nous désignerons cette population comme étant celle des entreprises «Net-Valorisantes »;

- la seconde population est composée des 174 PME dont les responsables n'ont pas constaté d'effet positif ni sur les gains de productivité ni sur la diversification; nous désignerons cette population comme étant celle des entreprises « Net-Indifférentes »;

L'enquête statistique a été réalisée selon une méthodologie présentée à l'annexe I.

\section{Valorisation d'Internet, appartenance sectorielle et équipements en TIC}

La notion de trajectoire technologique présentée précédemment peut être appréhendée par des caractéristiques telles que la nature sectorielle et technologique des PME des deux populations. Afin de relever l'existence de trajectoire technologiques différentes intervenant comme facteurs discriminants dans l'obtention d'avantages concurrentiels au regard des usages d'Internet et de TIC connexes, nous proposons une comparaison des tailles, des secteurs, des taux d'équipements, des dates de connexion à Internet ainsi que des réseaux informatiques et télécommutés existants dans les entreprises étudiées. 
Le tableau ci-après, précisant la nature des secteurs représentés dans les deux sous-populations, révèle (vu la stabilité entre les deux populations) que l'appartenance sectorielle ne peut pas constituer une base unique et solide d'explication des différences de valorisation d'Internet entre les deux sous-populations ${ }^{3}$.

TABLEAU 1

Les secteurs d'activité représentés

\begin{tabular}{lrrrr}
\hline & \multicolumn{2}{c}{ Net-Valorisantes } & \multicolumn{2}{c}{ Net-Indifférentes } \\
\hline Industries agroalimentaires & 9 & $4,9 \%$ & 13 & $7,5 \%$ \\
Services aux entreprises & 29 & $15,8 \%$ & 12 & $6,9 \%$ \\
Services aux particuliers & 9 & $4,9 \%$ & 10 & $5,7 \%$ \\
Éducation, santé, action sociale & 11 & $6 \%$ & 22 & $12,6 \%$ \\
Banques, assurances & & & & \\
$\quad$ et services financiers & 14 & $7,6 \%$ & 6 & $3,5 \%$ \\
Industries des biens de consommation & 25 & $13,7 \%$ & 13 & $7,5 \%$ \\
Industries des biens d'équipement & 14 & $7,6 \%$ & 12 & $6,9 \%$ \\
Industrie des biens intermédiaires & & & & \\
$\quad$ et énergie & 15 & $8,2 \%$ & 18 & $10,3 \%$ \\
Construction & 7 & $3,8 \%$ & 12 & $6,9 \%$ \\
Commerce de détail & 10 & $5,5 \%$ & 12 & $6,9 \%$ \\
Commerce de gros & 15 & $8,2 \%$ & 12 & $6,9 \%$ \\
Transports & 10 & $5,5 \%$ & 16 & $9,2 \%$ \\
Immobilier & 15 & $8,2 \%$ & 16 & $9,2 \%$ \\
\hline Total des répondants & 183 & & 174 & \\
\hline
\end{tabular}

Concernant les structures d'effectifs de ces PME, il apparaît une légère différence: les PME disposant des effectifs salariés les plus modestes sont plus représentées dans la population des Net-Valorisantes (les entreprises disposant d'effectifs salariés compris entre 10 et 50 employés étant alors plus concentrées dans la population des Net-Indifférentes). Si les écarts sont peu sensibles, il conviendra tout de même d'en tenir compte dans nos interprétations ultérieures.

3. Pour plus de précision, notons que la différence la plus importante entre les deux populations (moins de $9 \%$ toutefois) concerne le secteur des services aux entreprises auquel certaines recherches attribuent un rôle moteur dans la dynamique d'adoption d'Internet (Gadille et d'Iribarne, 2000). 
TABLEAU 2

Détails des effectifs*

\begin{tabular}{lcccc}
\hline & \multicolumn{2}{c}{ Net-Valorisantes } & \multicolumn{2}{c}{ Net-Indifférentes } \\
\hline 0 à 1 salariés & 39 & $21,3 \%$ & 21 & $12,1 \%$ \\
2 à 9 salariés & 78 & $42,6 \%$ & 67 & $38,5 \%$ \\
10 à 50 salariés & 66 & $36,1 \%$ & 86 & $49,4 \%$ \\
\hline
\end{tabular}

* Dans ce tableau ainsi que dans les suivants, les pourcentages ont été arrondis au dixième pour une meilleure lisibilité des résultats présentés. Par ailleurs, pour chaque variable étudiée, les tableaux reprendront l'architecture suivante : la $1^{\text {re }}$ colonne présente les différents items ou classes de la variable concernée (exemple: pour le tableau 1, les classes d'effectifs), les $2^{\mathrm{e}}$ et $4^{\mathrm{e}}$ colonnes indiquent respectivement les effectifs de chacune des deux populations en fonction des réponses obtenues alors que les $3^{\mathrm{e}}$ et $5^{\mathrm{e}}$ colonnes présentent les pourcentages correspondant à ces effectifs (respectivement pour la population des Net-Valorisantes et celle des Net-Indifférentes).

La date de connexion à Internet (et donc d'adoption de cette technologie) est un indicateur important, car les apprentissages par l'usage et la découverte des possibilités de nouveaux usages se constituent progressivement (Gadille et d'Iribarne, 2000b). L'analyse de ces dates révèle un léger décalage dans l'ancienneté des connexions qui est supérieure chez les Net-Valorisantes. Le temps écoulé après l'adoption de la technologie peut, dans une certaine mesure, être considéré comme l'une des bases de l'apprentissage et de la valorisation liés aux TIC en termes de productivité et de diversification.

TABLEAU 3

L'ancienneté de la connexion à Internet*

\begin{tabular}{lrrrr}
\hline & \multicolumn{2}{c}{ Net-Valorisantes } & \multicolumn{2}{c}{ Net-Indifférentes } \\
\hline Industries agroalimentaires & 9 & $4,9 \%$ & 13 & $7,5 \%$ \\
Moins de 1 an & 41 & $22,4 \%$ & 60 & $35,3 \%$ \\
Entre 1 an et 3 ans & 92 & $50,3 \%$ & 88 & $51,7 \%$ \\
Plus de 3 ans & 50 & $27,3 \%$ & 22 & $12,9 \%$ \\
\hline Total des répondants & 183 & \multicolumn{3}{c}{170} \\
\hline
\end{tabular}

* Par rapport à septembre 2001, date de l'enquête.

De même, les écarts moyens d'équipements informatiques, mesurés dans un premier temps par le nombre moyen d'ordinateurs, révèlent une tendance en faveur d'un équipement plus important pour les Net-Valorisantes. En outre, dans la mesure où l'obtention d'avantages concurrentiels peut être liée à la mise en réseaux informatiques ou télécommutés comme supports de services à valeur ajoutée, il nous a semblé important d'étudier les différences entre les deux populations en termes d'équipements en réseaux, Intranet, site Web et site Extranet. 
TABLEAU 4

Détail des équipements informatiques et des différents accès réseau*

\begin{tabular}{lcccc}
\hline & Net-Valorisantes & Net-Indifférentes \\
\hline $\begin{array}{l}\text { Nombre d'ordinateurs } \\
\quad\end{array}$ & 55 & $30,2 \%$ & 73 & $42,2 \%$ \\
de 1 à 2 ordinateurs & 82 & $45,1 \%$ & 69 & $39,9 \%$ \\
de 3 à 9 ordinateurs & 45 & $24,7 \%$ & 31 & $17,9 \%$ \\
$\quad$ plus de 10 ordinateurs & & & & \\
Dispose d'un... & 134 & $73,2 \%$ & 101 & $58,1 \%$ \\
$\quad$ (possibilité de plusieurs réponses) & 118 & $64,5 \%$ & 86 & $49,4 \%$ \\
$\quad$.. réseau informatique & 49 & $26,8 \%$ & 26 & $14,9 \%$ \\
$\quad$... site Web & 33 & $18,0 \%$ & 10 & $5,7 \%$ \\
$\quad$.. Intranet ou d'un groupware & .. site Extranet &
\end{tabular}

* Nous rappelons que la totalité des entreprises des deux populations comparées dans cet article est connectée à Internet.

La mesure de ces quatre indicateurs (réseau, site Web, Intranet, Extranet) montre une différence des équipements systématiquement en faveur des entreprises Net-Valorisantes (les écarts pouvant aller jusqu'à 15 points en pourcentage). Toutefois, cette tendance structurelle, si elle constitue un fait important, mérite d'être éclairée au moyen d'autres critères pour mieux différencier les contextes d'usages entre les deux populations. En nous appuyant sur le modèle d'analyse, nous mobilisons donc d'autres dimensions comme les capacités professionnelles et les modalités d'insertion dans un espace industriel.

\section{Capacités professionnelles et organisationnelles de la PME et usages d'Internet}

Les modalités d'usage, concernant à la fois le management et les utilisateurs en général, ne sont pas forcément homogènes et cohérentes dans l'entreprise, elles se distribuent selon des constructions professionnelles spécifiques des acteurs de l'organisation. Les capacités professionnelles et organisationnelles sont repérées à partir de plusieurs dimensions :

- le motif de la décision du responsable de l'entreprise quant à l'adoption d'Internet;

- les changements organisationnels (formels/informels) susceptibles de favoriser les usages d'Internet. L'occurrence de ces changements renseigne sur la nature de l'équilibre entre processus d'exploitation et d'exploration comme l'analyse March (1991);

- la proportion du personnel de l'entreprise dont l'ordinateur est connecté à Internet ; 
- la construction des compétences d'usage et de maintenance : l'entreprise s'est-elle donné les moyens en matière de formation pour alimenter les processus d'exploration et d'exploitation?

\subsection{Nature et contexte de prise de décision d'adopter Internet}

Afin de cerner le contexte en amont de la prise de décision et le degré d'intentionnalité dans l'adoption de la technologie, nous avons cherché à connaître le ou les motifs de la décision de connexion à Internet (en interrogeant les responsables des PME ou leurs proches collaborateurs).

TABLEAU 5

Motifs de la décision de la connexion à Internet (possibilité de plusieurs réponses)

\begin{tabular}{lcccc}
\hline Motifs & \multicolumn{2}{c}{ Net-Valorisantes } & \multicolumn{2}{c}{ Net-Indifférentes } \\
\hline Pour voir & 49 & $27 \%$ & 75 & $43,4 \%$ \\
$\begin{array}{l}\text { Demandé par un client, un fournisseur } \\
\quad \text { ou une autre entreprise }\end{array}$ & 51 & $28,2 \%$ & 31 & $17,9 \%$ \\
$\quad \begin{array}{l}\text { Dans le cadre d'un projet en vue } \\
\quad \text { d'applications précises }\end{array}$ & 66 & $36,5 \%$ & 42 & $24,3 \%$ \\
Autre & 35 & $19,3 \%$ & 36 & $20,8 \%$ \\
\hline
\end{tabular}

Même si les écarts ne dépassent pas 17 points de pourcentage (cas du motif de connexion «pour voir»), ces résultats appellent deux remarques générales.

\subsubsection{Stratégies d'adoption, gains de productivité, diversification de produits et usages d'Internet}

Les entreprises Net-Valorisantes se sont connectées dans des buts plus précis que les Net-Indifférentes. En effet, 36,5\% des Net-Valorisantes (contre 24,3\% des Net-Indifférentes) se sont connectées dans le cadre d'un projet en vue d'applications précises alors que seulement $27 \%$ des Net-Valorisantes se sont connectées à Internet «pour voir» (contre 43,4\% des Net-Indifférentes). Le fait que la mise en place des TIC s'intègre plus fréquemment dans un projet prédéfini pour les entreprises de la population des Net-Valorisantes semble rejoindre les conclusions de recherches récentes. Ainsi, Venkatraman (2000) cite la définition d'une vision stratégique comme la première des cinq étapes qu'il relève dans le cadre de développement de projet d'entreprise lié à Internet. Il met en perspective l'intérêt d'intégrer d'éventuelles utilisations d'Internet dans une stratégie d'entreprise globale et cohérente plutôt que de réaliser des actions isolées. Willcoks et Plant (2001) tirent les mêmes enseignements et précisent que les entreprises en retard sont restées focalisées sur

Revue internationale P.M.E., vol. 15, $\mathrm{n}^{\text {os }} 3-4,2002$ 
une vision (éventuellement technicienne) détachée de la vision stratégique de l'entreprise. Ces constats autorisent à penser que les entreprises Net-Indifférentes se sont lancées dans des développements liés à Internet (connexion et, parfois, création de site) sans avoir d'objectifs clairs ou véritables, mais peut-être, pour suivre certains concurrents, c'est-à-dire «pour faire comme les autres et y aller » (Amabile, Gadille et Messonier, 2000; Beatty, Shim et Jones, 2001).

Par ailleurs, soulignons tout de même que plus d'une PME sur quatre de la population des Net-Valorisantes ayant adopté Internet «pour voir» en bénéficie pleinement. Ce fait peut être interprété à partir de l'hypothèse d'une différence structurelle entre les deux populations exprimable en termes de capacités professionnelles et organisationnelles ainsi qu'en termes de modalités d'insertion dans des espaces industriels. On peut «faire comme les autres» et avoir ou ne pas avoir les capacités de valorisation selon le double critère de productivité et de diversification.

\subsubsection{Des différences de contraintes dans l'adoption d'Internet issues d'espaces industriels différenciés}

Le fait que les entreprises Net-Valorisantes se soient connectées plus pour répondre à la demande d'acteurs externes (fournisseurs, clients, autres, etc.) permet d'aller dans le sens de l'hypothèse d'une insertion des deux types de PME dans des espaces industriels où les contraintes ne sont pas identiques (Gadille et d'Iribarne, 2000). En outre, il est plausible de penser que les connexions effectuées à la demande d'un client, d'un fournisseur ou d'un autre type d'entreprise correspondent également à la concrétisation de projet précis (comme le basculement sur Internet d'échanges de données informatisés).

\subsection{Changements organisationnels et développement d'Internet}

L'investigation de l'hypothèse de capacités professionnelles et organisationnelles différenciées peut enfin être réalisée sur la base de questions liées aux changements d'organisation, à la diffusion de la technologie dans l'entreprise et aux politiques de formation.

De fait, $40 \%$ des entreprises Net-Valorisantes déclarent avoir modifié leur organisation lors du développement d'Internet contre seulement $8 \%$ des NetIndifférentes. En outre, relevons que les entreprises Net-Valorisantes sont plus utilisatrices de l'EDI ( $c f$. le tableau 9) et plus en réseau dans leur espace industriel (comme le montre le pourcentage de sites Extranet). Ainsi, dans une perspective d'analyse dynamique, on peut supposer que des changements organisationnels précédant l'adoption d'Internet ont déjà eu lieu dans cette population. Cette hypothèse permet d'éclairer le pourcentage relativement modeste ( $40 \%$ ) de répondants de cette population évoquant un changement organisationnel.

Revue internationale P.M.E., vol. 15, ns 3-4, 2002 
TABLEAU 6

Modification de l'organisation de l'entreprise lors du développement d'Internet

\begin{tabular}{lcccc}
\hline & \multicolumn{2}{c}{ Net-Valorisantes } & \multicolumn{2}{c}{ Net-Indifférentes } \\
\hline Pas d'accord, plutôt pas d'accord & 109 & $60 \%$ & 14 & $92 \%$ \\
D'accord, plutôt d'accord & 73 & $40 \%$ & 156 & $8 \%$ \\
\hline
\end{tabular}

La mise en évidence de la concomitance du changement organisationnel et de l'obtention d'avantages concurrentiels rejoint les résultats de recherches déjà anciennes (Eyraud, d'Iribarne et Maurice, 1988) comme de récentes conclusions propres aux technologies de l'Internet (Kalika, 2000).

De plus, les changements organisationnels peuvent être liés à une diffusion plus ou moins importante de la technologie dans l'organisation auprès de différentes catégories de salariés; c'est ce que révèle le test du khi carré présenté en annexe (tableau A1, annexe II). En effet, il semble exister une relation négative entre une distribution minoritaire des accès Internet auprès des salariés et le fait de modifier son organisation.

TABLEAU 7

Diffusion des NTIC dans l'entreprise : les acteurs ayant accès à Internet

\begin{tabular}{lcccc}
\hline & \multicolumn{2}{c}{ Net-Valorisantes } & \multicolumn{2}{c}{ Net-Indifférentes } \\
\hline La totalité ou la majorité du personnel & 99 & $55 \%$ & 49 & $29 \%$ \\
Seulement certaines personnes & 81 & $45 \%$ & 119 & $71 \%$ \\
\hline
\end{tabular}

À un premier niveau de lecture, nous remarquons que les entreprises déclarant valoriser Internet correspondent largement à celles ayant fait un effort pour rendre accessible et diffuser cette technologie. De fait, cette diffusion des NTIC à l'intérieur de l'organisation semble faciliter l'accès aux informations et soutenir l'interactivité dans certaines relations interpersonnelles (facilité à échanger des mails, des fichiers, etc.). Ces technologies peuvent donc être tenues pour l'un des facteurs favorisant, d'une part, la perméabilisation des frontières hiérarchiques ou fonctionnelles (Laval, 2000) et, d'autre part, l'élargissement des frontières organisationnelles (Benavent, 2000).

En outre, ces résultats prolongent les explications développées dans différentes recherches en management stratégique. En premier lieu, de nombreux travaux (Lesca, 1989; Marmuse, 1992 ; Martinet, 1991) rappellent que l'insuffisance des interactions et des échanges d'informations dans l'entreprise provient souvent de sa structure d'organisation. En effet, il paraît difficile d'entretenir une certaine

Revue internationale P.M.E., vol. 15, nos 3-4, 2002 
interactivité lorsque le degré de parcellisation ou de spécialisation des activités et le cloisonnement des informations en fonction des niveaux hiérarchiques et des fonctions sont importants (Eyraud, d'Iribarne et Maurice, 1988). Dans ce cas, le degré de diffusion d'Internet n'est qu'un reflet des structures professionnelles et de pouvoir dans l'entreprise favorisant sa performance. C'est ce que met en perspective le test du khi carré présenté en annexe II (tableau A4) concernant la relation entre les modifications de l'organisation et la conquête de nouveaux clients.

À l'inverse, le fait d'impliquer et d'aider, dès le départ, un ensemble important d'acteurs peut exercer une influence favorable sur la circulation des informations dans l'entreprise à condition de mettre en cohérence la formation avec l'évolution des règles organisationnelles, d'où l'intérêt de poser des questions sur les actions de formation.

TABLeau 8

Mise en place de ressources ou d'assistances lors de l'implantation et du développement des NTIC

\begin{tabular}{lccccc}
\hline & \multicolumn{2}{c}{ Net-Valorisantes } & \multicolumn{2}{c}{ Net-Indifférentes } \\
\hline $\begin{array}{l}\text { Organisation de formations } \\
\begin{array}{c}\text { Mise en place des assistances internes } \\
\text { pour les acteurs qui le souhaitaient }\end{array}\end{array}$ & 64 & $46,2 \%$ & 35 & $20,5 \%$ \\
\hline
\end{tabular}

Les résultats discutés dans cette section mettent en perspective certaines tendances qui différencient sensiblement la population des Net-Valorisantes de celle des Net-Indifférentes. La population des Net-Valorisantes comporte des PME dont les stratégies de connexion sont plus établies et plus contraintes par leurs relations industrielles. Les changements organisationnels sont également plus nombreux dans ces PME. De même, ces entreprises ont davantage investi en ce qui concerne la formation de leurs acteurs. Néanmoins, ces résultats ne permettent pas de conclure sur l'existence d'un lien précis entre des capacités organisationnelles plus importantes et le fait d'obtenir des gains de productivité ou de mettre en œuvre une diversification. En effet, le mode d'insertion de l'entreprise dans son espace industriel est une donnée complémentaire dans la recherche de variables discriminantes entre les deux populations. Il reste donc à étudier l'existence d'une relation entre la valorisation des usages d'Internet (par l'obtention de gains de productivité ou d'une diversification) et les modes d'insertion différenciés dans l'espace industriel pour les entreprises des deux populations. 


\section{Valorisation d'Internet et rapport de la PME à son espace industriel}

\subsection{Les applications utilisées comme révélateur de l'insertion dans un espace industriel différencié}

Dans un premier temps, le mode d'insertion de l'entreprise dans un espace industriel peut être appréhendé en analysant les types d'applications qu'elle mobilise pour médiatiser ses relations avec certains acteurs externes. Ces types d'applications (courrier électronique et échange de fichiers, veille, usage de l'EDI, etc.) nous renseignent sur la structure de l'usage de la technologie, mais également sur la structure probable des relations qui sous-tendent ces usages.

TABLEAU 9

Les applications développées (possibilité de plusieurs réponses)

\begin{tabular}{lrrrr}
\hline & \multicolumn{2}{c}{ Net-Valorisantes } & \multicolumn{2}{c}{ Net-Indifférentes } \\
\hline Échanger des mail et des fichiers & 158 & $86,8 \%$ & 122 & $70,9 \%$ \\
Rechercher des informations, & 157 & $86,3 \%$ & 131 & $76,1 \%$ \\
$\quad$ réaliser de la veille & 79 & $43,4 \%$ & 31 & $18 \%$ \\
Pratiquer l'EDI & 72 & $39,6 \%$ & 30 & $17,4 \%$ \\
Traiter des formulaires administratifs & 43 & $23,7 \%$ & 15 & $8,7 \%$ \\
Accéder à un Extranet & &
\end{tabular}

Concernant les différents types d'applications Internet, on observe un écart structurel entre les deux populations : avec des écarts oscillant entre 10 et 25 points de pourcentage, la population des Net-Valorisantes est plus utilisatrice que celle des Net-Indifférentes. On peut supposer que les entreprises Net-Valorisantes utilisent plus intensivement Internet pour, par exemple, gérer leurs relations dans leur espace industriel. C'est ce que révèle l'utilisation d'Internet pour traiter des formulaires administratifs ou pour pratiquer des échanges de données informatisés. On peut également supposer que les PME de cette population s'inscrivent dans des relations extérieures elles-mêmes porteuses d'une dynamique d'utilisation d'Internet. La différence du taux de PME utilisant Internet pour accéder à des Extranet reste très significative de ce point de vue: pratiquement une entreprise sur quatre dans la population des Net-Valorisantes contre moins de une entreprise sur dix dans la population des Net-Indifférentes. Les entreprises Net-Valorisantes apparaissent ainsi étroitement imbriquées dans les réseaux d'autres entreprises qui semblent avoir elles-mêmes des usages d'Internet soutenus. Dans le même temps, les entreprises de cette population font preuve d'une attention accrue à leur environnement comme le montrent les résultats suivants.

Revue internationale P.M.E., vol. 15, nos 3-4, 2002 
La mise en place de processus de veille ou de recherche d'informations sur Internet et la fréquence de ces processus constituent des variables permettant d'aborder les comportements de veille et d'attention des entreprises à leur environnement (Amabile, 1997).

TABLEAU 10

La fréquence d'usage d'Internet

pour rechercher des informations et / ou faire de la veille

\begin{tabular}{lrrcc}
\hline & \multicolumn{2}{c}{ Net-Valorisantes } & \multicolumn{2}{c}{ Net-Indifférentes } \\
\hline Régulièrement & 121 & $66,1 \%$ & 50 & $28,9 \%$ \\
Parfois & 52 & $28,4 \%$ & 84 & $48,5 \%$ \\
Jamais & 10 & $5,5 \%$ & 39 & $22,6 \%$ \\
\hline
\end{tabular}

On observe que, dans deux entreprises de la population Net-Valorisantes sur trois, les acteurs procèdent régulièrement à des recherches d'informations sur Internet alors que dans la population des Net-Indifférentes, cette proportion est inférieure à une entreprise sur trois. De plus, dans cette population, environ un quart des entreprises ne déclare pas de recherche d'informations contre seulement 5,5\% des Net-Valorisantes. Ces résultats mettent en évidence une différence importante de routines concernant la recherche d'information entre les deux populations. Cet écart pourrait correspondre aux capacités professionnelles des acteurs dans l'usage d'Internet. Ces capacités dans les PME Net-Valorisantes seraient alors construites sur la base d'un double mouvement :

- un mouvement de maîtrise accrue de la technologie afin de rester dans les réseaux organisés d'autres entreprises ou de pouvoir organiser soimême son réseau d'entreprises, d'agences ou d'établissements;

- un mouvement d'exploration de la technologie et, en particulier, d'Internet qui participe et soutient les processus d'innovation de la PME.

Nous proposons d'investir ces deux pistes respectivement dans les deux paragraphes suivants.

\subsection{Internet et les échanges avec des acteurs externes}

Les différences de structure d'usage d'Internet avec des acteurs externes peuvent être un révélateur d'espace industriel différencié entre nos deux populations. Dans le tableau suivant, pour l'ensemble des types d'acteurs externes cités, on observe que les PME Net-Valorisantes sont sensiblement plus nombreuses que les Net-Indifférentes pour communiquer par Internet. Ainsi, les organisations utilisant Internet avec des entreprises partenaires représentent $57,4 \%$ des entreprises Net-Valorisantes contre seulement $24,4 \%$ des Net-Indifférentes. Ce résultat 
souligne la pertinence de l'hypothèse de rapports à l'environnement différents entre les deux populations: les entreprises Net-Valorisantes seraient plus construites sur des relations partenariales que les Net-Indifférentes. À cette hypothèse s'ajoute celle d'une diffusion de la technologie plus importante dans l'espace industriel des Net-Valorisantes.

TABLEAU 11

Les acteurs externes avec lesquels les PME utilisent les NTIC (possibilité de plusieurs réponses)

\begin{tabular}{lcccc}
\hline & \multicolumn{2}{c}{ Net-Valorisantes } & \multicolumn{2}{c}{ Net-Indifférentes } \\
\hline Clients & 151 & $82,5 \%$ & 94 & $57,3 \%$ \\
Fournisseurs & 137 & $74,9 \%$ & 94 & $57,3 \%$ \\
Établissements, agences, siège & 74 & $40,5 \%$ & 33 & $20,1 \%$ \\
Entreprises partenaires & 105 & $57,4 \%$ & 40 & $24,4 \%$ \\
Banque, comptable & 102 & $55,8 \%$ & 60 & $36,6 \%$ \\
CCI & 62 & $33,9 \%$ & 32 & $19,5 \%$ \\
Collectivités territoriales & 33 & $18 \%$ & 10 & $6,1 \%$ \\
Services administratifs & 52 & $28,4 \%$ & 23 & $14 \%$ \\
\hline
\end{tabular}

\subsection{Les avantages de l'exploration sur Internet}

Il est possible d'apporter des éléments complémentaires dans la construction de l'hypothèse d'un comportement plus exploratoire pour les PME Net-Valorisantes. Pour cela, il a été demandé aux responsables s'ils avaient pu trouver de nouveaux fournisseurs, de nouveaux partenaires ou de nouveaux clients en utilisant Internet et si cette technologie leur avait permis d'améliorer la qualité des produits ou services.

Les résultats obtenus à la première série de questions révèlent qu'environ la moitié des PME Net-Valorisantes ont constaté que leur usage d'Internet avait un impact positif sur leur recherche de nouveaux clients, fournisseurs et partenaires. De plus, les écarts entre les proportions de PME Net-Indifférentes et NetValorisantes sur chacun de ces items sont relativement importants (de 31 à 44 points de pourcentage).

Ce résultat montre la justesse des remarques précédentes sur un usage plus intensif d'Internet dans les processus d'exploration au sein des entreprises NetValorisantes. De plus, le test du khi carré, congruent avec ce résultat, révèle qu'il existe une relation positive entre le fait de distribuer la technologie d'Internet à une totalité ou à une majorité de salariés et le fait de trouver de nouveaux clients (tableau A2, annexe II). De même, il existe une relation positive entre une distribution généralisée de l'accès Internet aux salariés de l'entreprise et le fait de trouver de nouveaux partenaires (tableau A3, en annexe II).

Revue internationale P.M.E., vol. 15, $\mathrm{n}^{\text {os }} 3-4,2002$

(C) 2003 - Presses de l'Université du Québec

Édifice Le Delta I, 2875, boul. Laurier, bureau 450, Sainte-Foy, Québec G1V 2M2 • Tél. : (418) 657-4399 - www.puq.uquebec.ca

Tiré de : Revue internationale P.M.E., vol. 15, nos 3-4, sous la direction de Pierre-André Julien. 
Il semble que ces pratiques viennent alimenter des processus d'innovation au sein desquels le partenariat et le fonctionnement en réseau permettent de mutualiser ou de mettre en complémentarité certaines ressources.

TABLEAU 12

L'impact d'Internet sur la connaissance de nouveaux fournisseurs, partenaires et sur l'élargissement de la clientèle

L'utilisation d'Internet a permis

à votre entreprise de...
... trouver de nouveaux fournisseurs.

Net-Valorisantes Net-Indifférentes

Pas d'accord, plutôt pas d'accord

D'accord, plutôt d'accord

$\begin{array}{rrrrr}70 & 39 & \% & 141 & 82,5 \% \\ 109 & 61 & \% & 30 & 17,5 \%\end{array}$

... connaître de nouvelles entreprises

partenaires

Pas d'accord, plutôt pas d'accord

D'accord, plutôt d'accord

$53,4 \%$

$83 \quad 46,6 \%$

$15490,1 \%$

... d'élargir votre portefeuille clientèle

Pas d'accord, plutôt pas d'accord

$\begin{array}{llll}92 & 52,9 \% & 140 & 84,4 \%\end{array}$

D'accord, plutôt d'accord

$82 \quad 47,1 \% \quad 26 \quad 15,6 \%$

Les résultats obtenus en matière d'amélioration de la qualité à partir d'usage d'Internet révèlent une différence d'impact très importante entre les deux populations. Il apparaît que l'écart concernant la proportion des entreprises ayant amélioré la qualité de leurs services ou de leurs produits grâce à leur utilisation des NTIC est de 58 points en faveur des entreprises Net-Valorisantes. Plus largement, ce résultat vient corroborer l'hypothèse selon laquelle les différentes sources d'avantages concurrentiels peuvent être combinées au sein d'une même organisation selon des contextes organisationnels et industriels spécifiques.

TABLEAU 13

Utilisation des NTIC et amélioration de la qualité des services ou de produits

\begin{tabular}{lrrrr}
\hline & \multicolumn{2}{c}{ Net-Valorisantes } & \multicolumn{2}{c}{ Net-Indifférentes } \\
\hline Pas d'accord, plutôt pas d'accord & 43 & $23,7 \%$ & 139 & $81,3 \%$ \\
D'accord, plutôt d'accord & 138 & $76,3 \%$ & 32 & $18,7 \%$ \\
\hline
\end{tabular}




\section{Conclusion}

L'économie de la flexibilité fait peser deux exigences contradictoires sur les PME : une exigence de réduction des coûts de production et une exigence de capacité d'innovation dans les processus de production, dans les produits ou les services. Il semble désormais acquis que la recherche de ces deux types d'avantages concurrentiels peut être soutenue sous certaines conditions par l'usage d'Internet et de ses technologies connexes. Dans cet article, nous nous sommes donc intéressés aux caractéristiques des PME capables de réaliser des gains de productivité et de mettre en œuvre une diversification de leurs produits ou services à partir de leurs usages d'Internet. Les notions de trajectoire technologique, de capacités professionnelles et organisationnelles ainsi que celles d'espace d'industriel ont été mobilisées pour caractériser le contexte d'usage d'Internet favorable à l'obtention de ces deux types d'avantages concurrentiels. Les résultats principaux indiquent qu'il existe une distinction structurelle entre des PME capables à la fois de réaliser des gains de productivité et de se diversifier par l'usage d'Internet et des PME qui n'obtiennent ni gain de productivité ni diversification par cet usage. En premier lieu, ces distinctions structurelles portent moins sur leur équipement en TIC de base (ordinateurs et réseaux informatiques) que sur les équipements en réseaux porteurs de services à valeur ajoutée (site Web, site Extranet, Intranet et «collecticiel»). En deuxième lieu, elles portent sur des capacités de changements organisationnels et professionnels plus importantes au sein de la population des PME innovantes et bénéficiant de gains de productivité. Ces différences structurelles apparaissent liées aux modalités d'insertion de ces PME dans un espace industriel spécifique. Cet espace industriel se caractérise par des relations de travail et d'échange dans des réseaux au sein desquels l'usage Internet apparaît comme dominant à la fois dans les processus d'exploitation et les processus d'exploration. En dernier lieu, on peut supposer que les processus d'exploration soutenus par l'usage d'Internet permettent de pallier la perte d'autonomie de la PME intégrée verticalement dans le réseau de la grande entreprise et de rester acteur de sa viabilité à moyen et long terme.

Revue internationale P.M.E., vol. 15, nos 3-4, 2002 


\section{ANNEXE I \\ Méthodologie de l'enquête}

Le questionnaire sur lequel repose l'enquête et les résultats dont nous rendons compte dans ce papier a été administré en septembre 2001, par voie téléphonique sur une population de 2223 établissements de la région Provence-Alpes-Côte d'Azur.

La constitution de la population à enquêter a été réalisée sur la base de la méthode des quotas, garantissant un effectif identique et significatif selon tous les secteurs d'activité. La structure par taille et secteur est composée comme suit: 4 tranches d'effectif salarié et 13 secteurs balayant l'ensemble des activités économiques. À partir de fichiers sources provenant des chambres de commerce et d'industrie de la région, un tirage au sort a été effectué dans chacune de ces souspopulations au fur et à mesure de la réalisation des entretiens téléphoniques. Il faut noter que, sur cette population enquêtée, $86,5 \%$ des établissements ont moins de 50 salariés. Cet ensemble d'établissement se décompose de la façon suivante:

- 16,3\% d'établissements de 0 à 1 salarié ;

- $36 \%$ d'établissements de 2 à 9 salariés ;

- 34, $2 \%$ d'établissements de 10 à 50 salariés.

Les PME des deux populations étudiées dans cet article sont extraites de cette enquête selon les modalités suivantes :

- il s'agit d'établissements de moins de 50 salariés;

- ce sont tous des sièges sociaux, dont certains peuvent posséder d'autres établissements ;

- les répondants sont toujours des responsables de l'entreprise, soit la direction générale, l'adjoint à la direction générale ou le gérant, soit l'un de ses proches collaborateurs (directeur administratif ou financier, directeur informatique). 


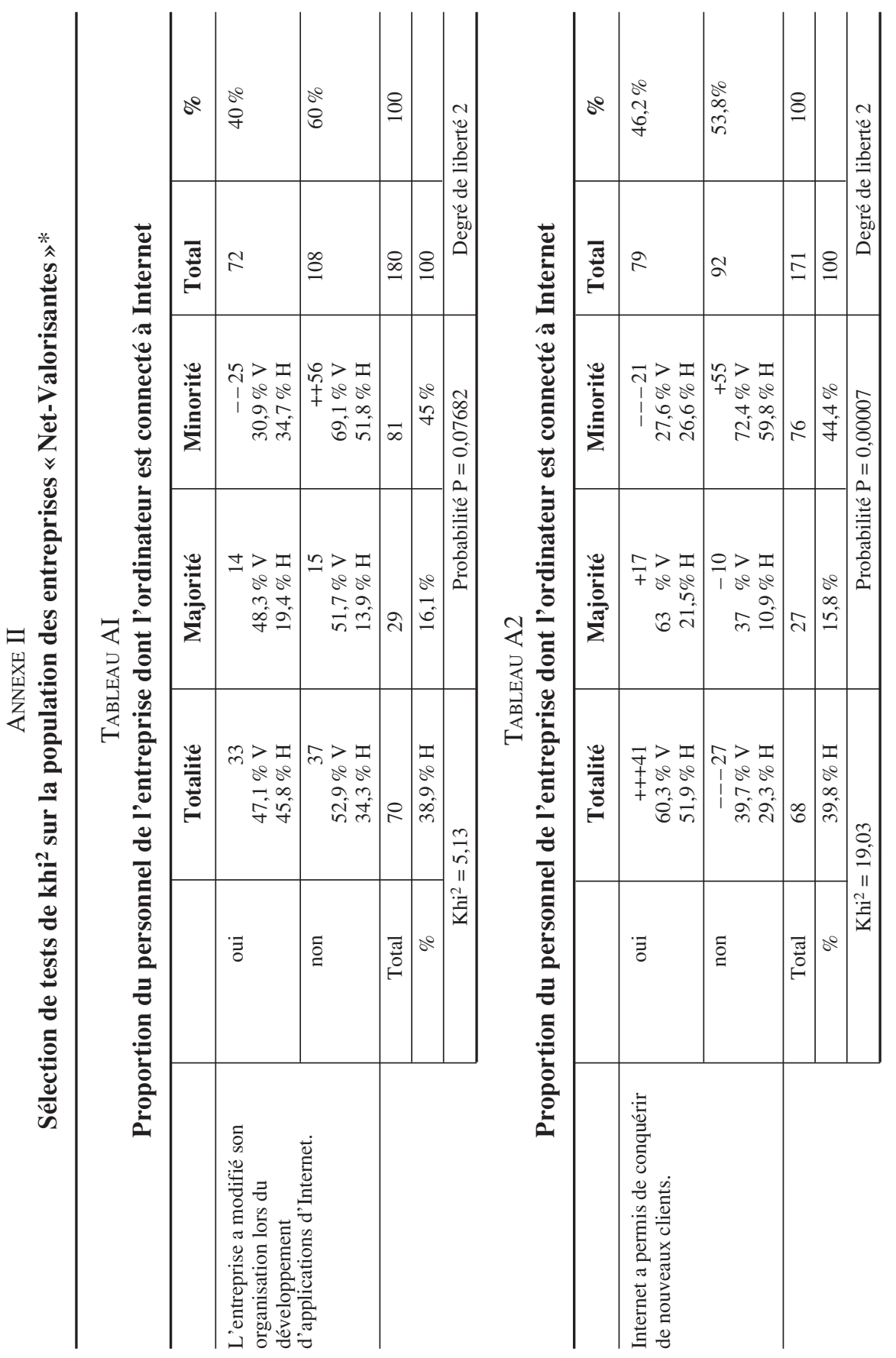

Revue internationale P.M.E., vol. 15, nos 3-4, 2002 


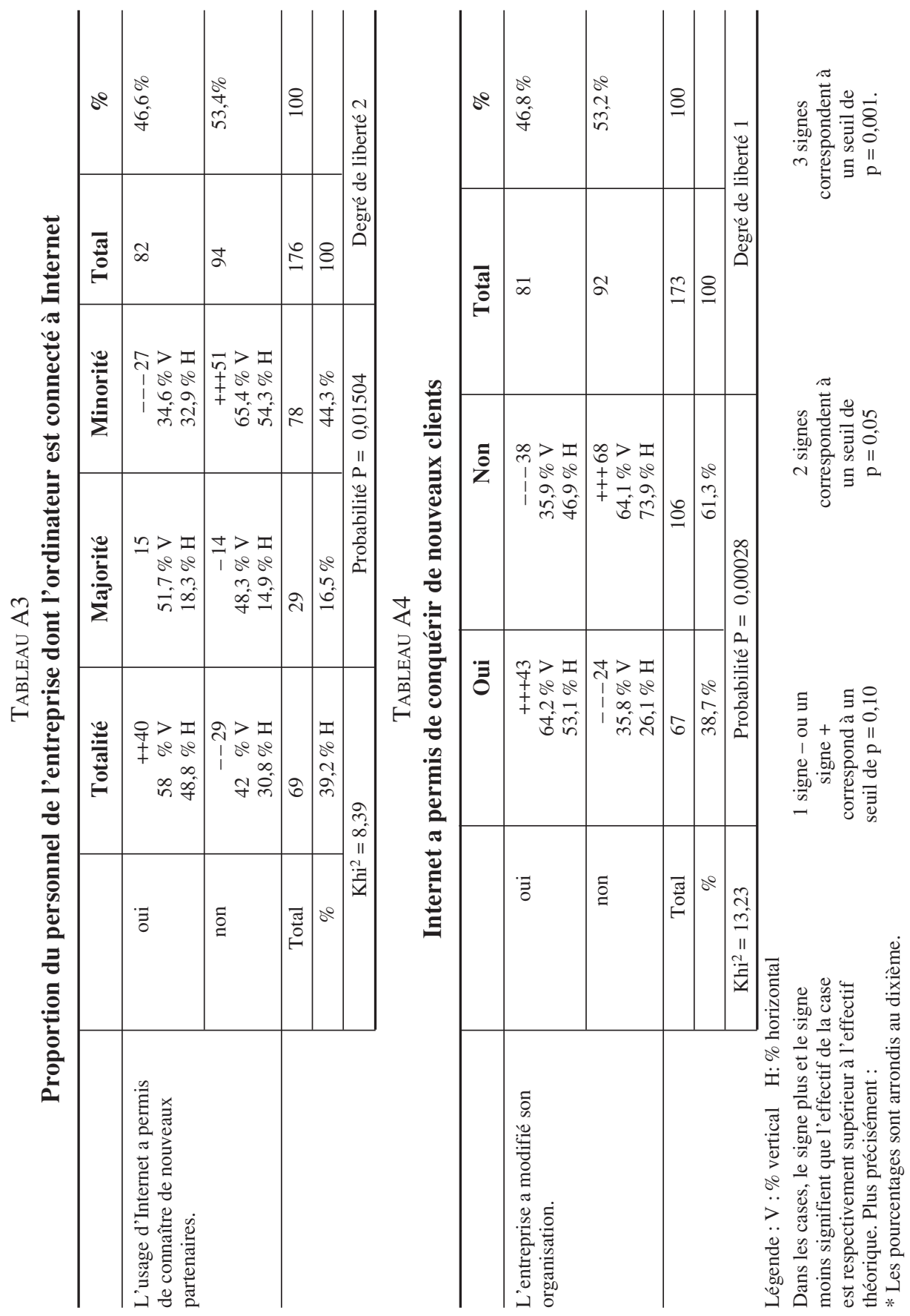

Revue internationale P.M.E., vol. 15, nos 3-4, 2002 


\section{Bibliographie}

AmABILE, S. (1997), Contribution à l' ingénierie de l'organisation : de la veille stratégique à l'attention organisationnelle. Illustration: le réseau d'attention des mutuelles niortaises, Thèse de doctorat en sciences de gestion, GRASCE, Université d'AixMarseille III.

AMABILE, S., M. GADILLE et R. MessonieR (2000), «Information, organisation, décision : étude empirique sur les apports des NTIC dans des PME "Internautes" », Systèmes d'information et management, Paris, vol. $5, \mathrm{n}^{\circ} 1$.

AMABILE, S. et M. GADILLE (2003), «Les NTIC dans les PME : Stratégies, capacités organisationnelles et performances différenciées », Revue française de gestion (à paraître).

BEATTY, R.C., J.P. SHIM et M.C. JONES (2001), « Factors influencing corporate Web site adoption : a time-based assessment », Information \& Management, vol. 38, p. 337-354.

BENAVENT, C. (2000), «Les NTIC, le marketing stratégique et le jeu concurrentiel», Revue française de gestion, $\mathrm{n}^{\circ}$ 129, p. 91-100.

CHANDLER A.D. (1992), «Organizational capabilities and the economic history of the industrial entreprise », Journal of Economic Perspectives, vol. 6, nº 3, p. 79-100.

EYRAUD, F., A. D'IRIBARNE et M. MAURICE (1988), « Des entreprises face aux technologies flexibles: une analyse de la dynamique du changement», Sociologie du travail, $\mathrm{n}^{\mathrm{o}} 1, \mathrm{p} .55-77$.

GADILle, M. et S. AmABILE (2002), L'usage des technologies de l'information et de la communication par les entreprises en Provence Alpes-Côte d'Azur, Rapport de recherche réalisé dans le cadre d'un dispositif régional d'observation de la diffusion des TIC coordonné par le Conseil régional, la Chambre régionale de commerce et d'industrie de Provence-Alpes-Côte d'Azur, Corse, juin, 146 p.

GAdille, M. et A. D'IrIBARNE, 2000a, « Développement et Internet. Connaître pour agir », Guide à l'usage des acteurs intéressés par les politiques territoriales de diffusion des TIC dans les PME (réalisé dans le cadre du Programme d'initiative communautaire ADAPT), $56 \mathrm{p}$.

GADILLE, M. et A. D'IRIBARNE (2000b), « La diffusion d'Internet dans les PME : motifs d'adoption dans les réseaux et ressources mobilisées », Réseaux, n 104, vol. 18.

GADILlE, M. et A. D’IRIBARNE (2000c), «Les PME françaises et Internet », FormationEmploi, $\mathrm{n}^{\circ} 72$, octobre-décembre.

KALIKA, M. (2000), «Le management est mort, vive le e-management », Revue française de gestion, $\mathrm{n}^{\circ} 129$, juin-juillet-août, p. 68-74.

LAVAL, F. (2000), «Gestion des ressources humaines et NTIC : enjeux et perspectives », Revue française de gestion, $\mathrm{n}^{\circ} 129$, juin-juillet-août, p. 80-90.

LESCA, H. (1989), Information et adaptation de l' entreprise, Paris, Masson.

LEVITT, B. et J.G. MARCH (1988), «Organizational learning », Annual Review of Sociology, vol. 14, p. 319-340.

Revue internationale P.M.E., vol. 15, nos 3-4, 2002 
MARCH, J.G. (1991), «Exploration and exploitation in organizational learning», Organization Science, vol. 2, $\mathrm{n}^{0}$ 1, p. 71-87.

Marmuse, C. (1992), Politique générale. Langages, intelligence, modèles et choix stratégiques, Paris, Economica.

MARTINET, A.-C. (1991), «Management en temps réel et continuité stratégique sont-ils compatibles? », Revue française de gestion, novembre-décembre.

NELSON, R.R. (1991), «Why firms differ, and how does it matter?», Strategic Management Journal, vol. 12, p. 61-74.

NELSON, R.R. et S.G. WinTER (1982), An Evolutionary Theory of Economic Change, Cambridge, Harvard University Press.

PORTER, M. (1985), Competitive Advantage : Creating and Sustaining Superior Performance, NewYork, The Free Press, Macmillan.

Rogers, E. M. (1983), Diffusion of Innovation, $3^{\mathrm{e}}$ édition, New York, Free Press.

RosenBERG, N. (1982), «Learning by using », dans N. Rosenberg, Inside the Black Box, Cambridge, Cambridge University Press.

TEECE, D. et G. PISANO (1998), «The dynamic capabilities of the firms : an introduction », dans G. Dosi, D.J. Teece, et J. Chitry (dir.), Technology, Organization, and Competitiveness, Oxford et New York, Oxford University Press, p. 193-212.

VENKATRAMAN, N. (2000), «Five steps to a dot-com strategy: how to find your footing on the Web », Sloan Management Review, printemps, vol. 41, no 3, p. 15-28.

WillCoKs, L.P. et R. PlAnT (2001), «Pathways to E-business leadership : getting from bricks to clicks », Sloan Management Review, printemps, vol. 42, no 3, p. 50-59.

Revue internationale P.M.E., vol. 15, $\mathrm{n}^{\text {os }} 3-4,2002$ 